\title{
The $p+{ }^{9} \mathrm{Be}$ (thin target) reaction as a source of quasi-monoenergetic neutrons
}

\author{
J. Novák ${ }^{1}$, P. Bém ${ }^{1}$, M. Majerle ${ }^{1}$, J. Mrázek ${ }^{1}$, E. Šimečková ${ }^{1}$, M. Štefánik ${ }^{1}$, and Z. Yasin ${ }^{1,2}$ \\ 1 Nuclear Physics Institute of The Academy of Sciences of the Czech Republic, 25068 Řež 130, Czech Republic \\ 2 Physics Division, PINSTECH, P.O. Nilore, Islamabad
}

\begin{abstract}
The cyclotron driven fast quasi-monoenergetic neutron source based on the $\mathrm{p}+{ }^{9} \mathrm{Be}$ (thin target) reaction was studied at the proton energy around $30 \mathrm{MeV}$ Due to higher melting point of $\mathrm{Be}$, the $\mathrm{p}+{ }^{9} \mathrm{Be}(\mathrm{thin})$, reaction could be considered as an alternative to the most used $\mathrm{p}+\mathrm{Li}$ (thin) neutron source, providing a similar quasi-monoenergetic neutron spectrum at significantly higher neutron output owing to advance in higher incident proton beam current. The neutron spectrum measured by the time-of-flight method agrees with other experimental data and indicates dominant contribution of ground and first excited states leading to only two peaks, separated by some $2-3 \mathrm{MeV}$, in the $\mathrm{p}+\operatorname{thin}{ }^{9} \mathrm{Be}$ neutron spectrum.
\end{abstract}

\section{Introduction}

Neutron cross-sections (CS) at energies higher than few $\mathrm{MeVs}$ are becoming increasingly important in the future energy production. The experimentally measured CS data for neutrons with energies above some $20 \mathrm{MeV}$ are rare. In parallel with the experimental work, the need for neutron data at higher energies is reflected in the extensions of the commonly used libraries to higher energies.

Irradiation with the known neutron spectrum and the subsequent activity measurement is commonly used to determine the reaction CS. Only quasi-monoenergetic (QM) beams are available for neutron energies over $20 \mathrm{MeV}$. The $\mathrm{p}+\mathrm{Li}$ reaction is the most used QM neutron generator.

A significant disadvantage of the $\mathrm{p}+\mathrm{Li}$ neutron generator presents the low melting point temperature $\left(180.5^{\circ} \mathrm{C}\right)$, which limits the maximum proton current directed to $2-5 \mathrm{~mm}$ thick Li foils to around $10 \mu \mathrm{A}$. A good replacement might be the Be material (the melting point of $\mathrm{Be}$ is $1287^{\circ} \mathrm{C}$ ). The cost of such advantage are worse quasi-monoenergetic properties of the neutron spectrum.

The reaction $\mathrm{p}+\mathrm{Be}$ was studied with the time-offlight (TOF) technique already in seventies at different laboratories $[1,2]$. Two close peaks were observed in the neutron spectrum.

Uwamino measured the TOF spectrum of the $\mathrm{p}+\mathrm{Be}$ reaction on 1 and $2 \mathrm{~mm}$ thick Be targets at energies 20, $25,30,35$ and $40 \mathrm{MeV}$ [3]. He proved the usability of such spectrum for CS measurements by the activation method for the $\mathrm{Al}$ and Au nuclei. J.H.Kim et al. have used $0.5 \mathrm{~mm}$ thick foil and $35 \mathrm{MeV}$ proton beam to measure the $\mathrm{p}+\mathrm{Be}$ TOF spectrum [4]. They have obtained a neutron spectrum with well separated peaks to ground and excited states of the ${ }^{9} \mathrm{~B}$.

The usability of the $\mathrm{p}+\mathrm{Be}$ reaction at 79.4 and $118 \mathrm{MeV}$ proton energy for cross-section measurements was confirmed in the work of J.Sisterson at iThemba
Lab [5]. Zaman et al. have used the neutrons produced on $5 \mathrm{~mm}$ thick Be target with 25,35 and $45 \mathrm{MeV}$ protons to measure the cross-sections of ${ }^{89} \mathrm{Y}(\mathrm{n}, 2-4 \mathrm{n})$ reaction and have obtained good agreement with existing experimental data [6].

The evaluation of the proton library included in ENDF/B-VII was used to calculate the $\mathrm{p}+\mathrm{Be}$ and $\mathrm{p}+\mathrm{Li}$ spectra using MCNPX code, Fig. 1. The predicted $\mathrm{p}+\mathrm{Be}$ neutron spectrum has several monoenergetic peaks, and is as such not suitable as a source of QM neutrons. This evaluation is in disagreement with the mentioned experimental data.

It seems that the $\mathrm{p}+\mathrm{Be}$ reaction could provide a $\mathrm{QM}$ neutron spectrum similar to the spectrum from the $\mathrm{p}+\mathrm{Li}$ reaction and significantly higher neutron outputs. The slightly wider monoenergetic peak from the $\mathrm{p}+\mathrm{Be}$ reaction would be a good price for a much larger scale of potential isotopes, which could be produced in the higher neutron flux.

With the present status of things, we decided to measure the neutron spectrum from the $\mathrm{p}+\mathrm{Be}$ reaction at the proton energy around $30 \mathrm{MeV}$ and to study the different aspects of using the $\mathrm{p}+\mathrm{Be}$ reaction as the source of the QM neutrons.

\section{Experimental setup}

The protons from the cyclotron U120M accelerated to $30 \pm 0,5 \mathrm{MeV}$ with the current $1 \mu \mathrm{A}$ were focused and directed to the $0.5 \mathrm{~mm}$ thick Be target backed with the $10 \mathrm{~mm}$ graphite beam stopper. Around $3 \mathrm{~m}$ from the neutron production target, a neutron shielding made of $50 \mathrm{~cm}$ borated polyethylene with the $6 \mathrm{~cm}$ square shape hole was installed (Fig. 2). The collimated beam was stopped in the $50 \mathrm{~cm}$ block of borated polyethylene installed around $17 \mathrm{~m}$ from the target. The axis of the neutron beam is identical with the axis of the proton beam. 


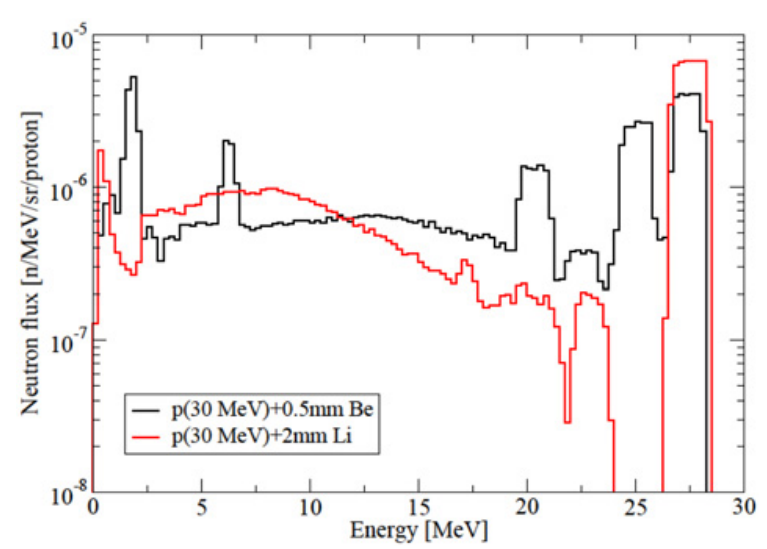

Figure 1. MCNPX (ENDFB-VII and LA150 libraries were used) simulated neutron spectra from the reactions $\mathrm{p}+\mathrm{Be}$ and $\mathrm{p}+\mathrm{Li}$ with $30 \mathrm{MeV}$ protons.

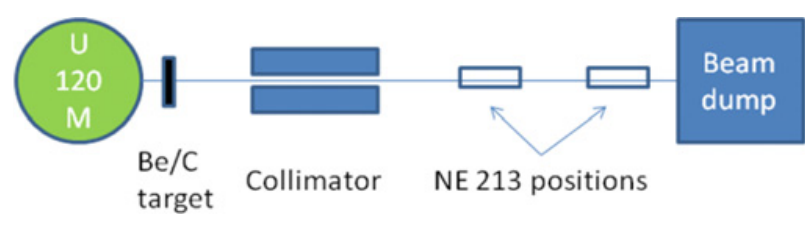

Figure 2. Scheme of the experimental setup.

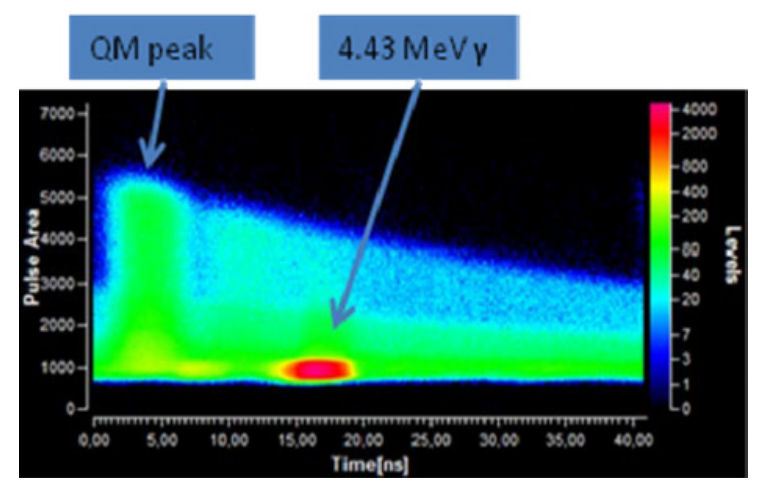

Figure 3. Pulse height vs. pulse phase data collected from the thin Be target with the NE213.

We measured the neutron spectra in the TOF mode at three distances from the target: $3.2 \mathrm{~m}, 10.0 \mathrm{~m}$, and $15.0 \mathrm{~m}$. The signals from the anode of the $2^{\prime \prime} \times$ 2" NE213 scintillator probe were recorded with the CAEN V1751 digitizer, another channel of the digitizer was simultaneously sampling the cyclotron accelerating frequency (RF).

Neutron and gamma pulses were separated by the pulse shape analysis. The relation between time tags of pulses and time of the RF phase served for TOF determination (Fig. 3). The $4.43 \mathrm{MeV}$ gammas, generated in the reaction of proton with the carbon stopper simultaneously with neutrons, were used as the time reference to determine the neutron speed.

The time resolution corresponds to time FWHM of the peak of $4.43 \mathrm{MeV}$ gammas.

The NE213 scintillator efficiency was calculated with the dynamic threshold method. The method operates only with protons scattered to limited angular band. This approach removes the necessity to know the whole response function of the scintillator, the results are based

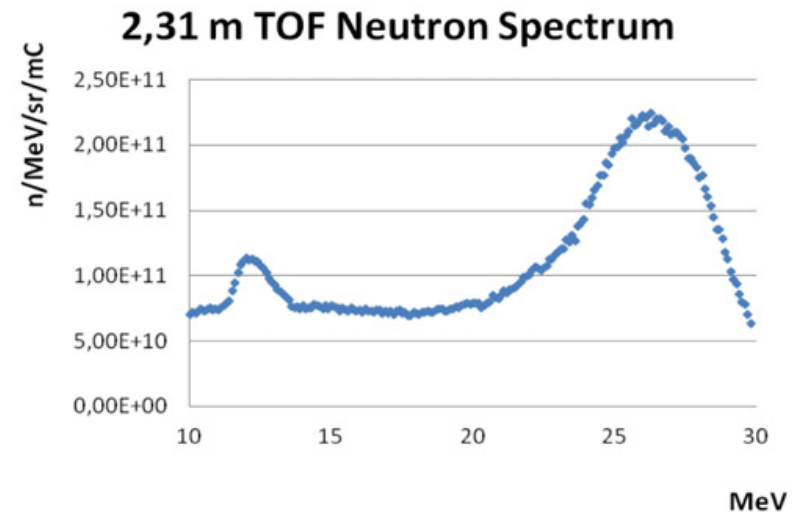

Figure 4. Neutron spectrum obtained for the target-detector distance $2.31 \mathrm{~m}$.

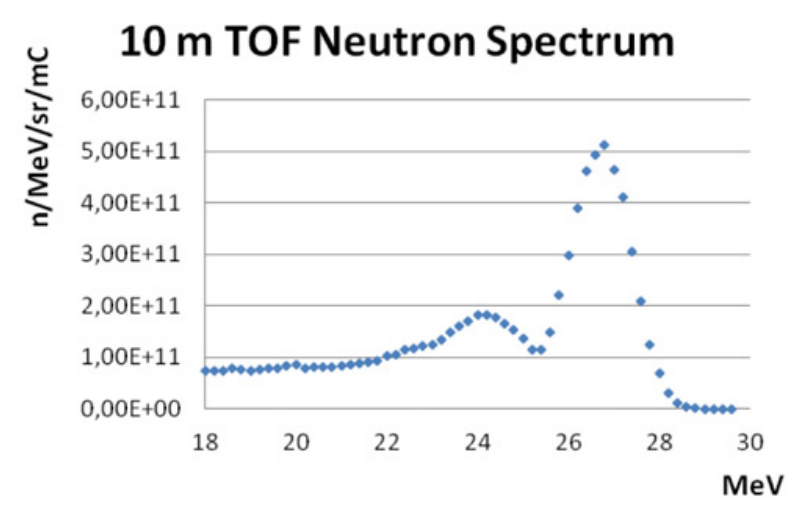

Figure 5. Neutron spectrum obtained for the target-detector distance $10.0 \mathrm{~m}$.

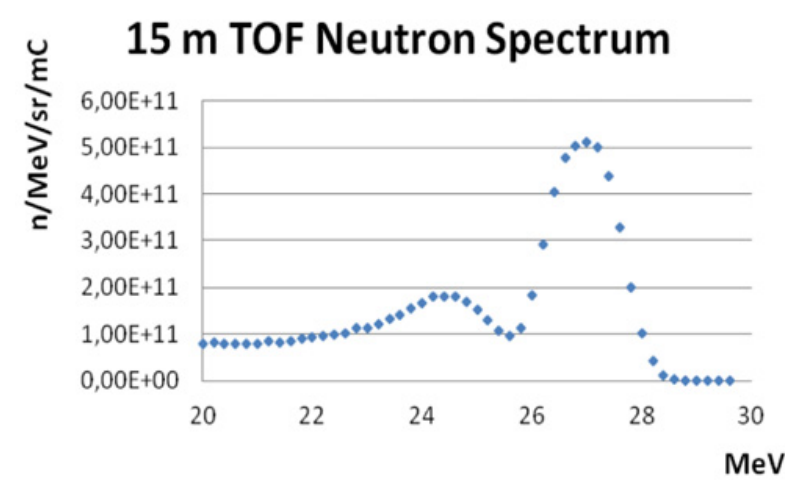

Figure 6. Neutron spectrum obtained for the target-detector distance $15.0 \mathrm{~m}$.

only on the cross-section of elastic scattering of neutrons on hydrogen [7].

\section{Time-of-flight results}

The neutron spectra obtained by the TOF method are shown in the Figs. 4-6.

The target-detector distance $2.31 \mathrm{~m}$ covers the largest energy range from $30 \mathrm{MeV}$ down to $15 \mathrm{MeV}$. Below this energy, a peak of gammas which could not be perfectly separated is visible. At short base, the high intensity of neutron and gamma field leads to pile-up of pulses. It deteriorates the shape of pulses with low amplitude and the n-gamma discrimination of lower pulses does not 
work. The detailed structure of the spectrum is not seen due the TOF spread about $3 \mathrm{~ns}$ and relatively strong time dependence on the energy at short base.

For the longer base, the energy resolution at the same time resolution is better due the lower velocity determination error, but the energy range is narrower due the time frame overlap.

At 10 and $15 \mathrm{~m}$, the time resolution allowed to see neutron peaks coming from ground and excited states.

\section{Conclusions}

The results from our measurements are in good agreement with the Korean measurements [4] and indicate only two peaks, separated by some $2-3 \mathrm{MeV}$. There are no other peaks observed above $18 \mathrm{MeV}$. Simulated spectra from Be target need to eliminate the neutron peaks from higher excited states. Without further peaks in this energy range, the thin Be target can be considered as a replacement for the thin Lithium target in QM neutron generator.

The spectrum is influenced also by the neutron interaction on the target station material, including the Carbon backing. Therefore, the measurement and the simulation of the neutron spectra at all energy range of protons are necessary.

The calculation in SRIM shows that in $1 \mathrm{~mm}$ thick Be $35 \mathrm{MeV}$ protons should have energy losses about $2.5 \mathrm{MeV}$, which should be enough for the peaks to overlap and provide one, well defined neutron peak.

Some drawback of the $\mathrm{p}+\mathrm{Be}$ reaction consists in the determination of monoenergetic peak intensity, which can't be determined by the target activity measurement, as it can be determined in the $\mathrm{p}+\mathrm{Li}$ case [8]. However, in practice most laboratories rely on direct or indirect (proton current measurement) methods of monitoring the integral neutron flux. There is also no information at present on the lower energy part of the neutron spectrum. The up-todate measurements have only excluded significant increase of the neutron flux for energies above some Ep - $15 \mathrm{MeV}$.
The possible neutron peaks below this limit could make the extraction of CS from such complex neutron spectra very complicated.

\section{References}

[1] J. Jungerman, Production of medium-energy neutrons from proton bombardement of light elements, Nuclear Instruments and Methods 94, 421-427 (1971)

[2] J. Watson, The neutron time-of-flight facility at the Manitoba cyclotron laboratory, Nuclear Instruments and Methods 164, 129-141 (1979)

[3] Y. Uwamino, Semi-monoenergetic neutron field for activation experiments up to $40 \mathrm{MeV}$, Nuclear Instruments and Methods in Physics Research A 271, 546-552 (1988)

[4] J.H. Kim, A measurement of monoenergetic neutrons from ${ }^{9} \mathrm{Be}(\mathrm{p}, \mathrm{n}){ }^{9} \mathrm{~B}$, Journal of the Korean Physical Society 32(4), 462-467 (1998)

[5] J. Sisterson, Cross-section measurements for neutron-induced reactions in copper at neutron energies of 70.7 and $110.8 \mathrm{MeV}$, Nuclear Instruments and Methods in Physics Research B 240, 617-624 (2005)

[6] M. Zaman, Measurement of cross-sections for ${ }^{89} \mathrm{Y}(\mathrm{n}, \mathrm{xn})$ reaction at average neutron energies of $15-36 \mathrm{MeV}$, Journal of Radioanalytical and Nuclear Chemistry 303, 815-820 (2015)

[7] M. Majerle, $\mathrm{Au}, \mathrm{Bi}, \mathrm{Co}$ and $\mathrm{Nb}$ cross-section measured by quasimonoenergetic neutrons from $\mathrm{p}+{ }^{7} \mathrm{Li}$ reaction in the energy range of $18-36 \mathrm{MeV}$, Nuclear Physics A 953, 139-157 (2016)

[8] S. Schery, Activation and angular distribution measurements of ${ }^{7} \mathrm{Li}(\mathrm{p}, \mathrm{n})^{7} \mathrm{Be}(0.0+0.429 \mathrm{MeV})$ for $\mathrm{E}_{\mathrm{p}}=25-45 \mathrm{MeV}$ : A technique for absolute neutron yield determination, Nuclear Instruments and Methods 147, 399-404 (1977) 